\title{
MEASURING THE QUALITY OF AIR TRANSPORT NETWORKS
}

Bert Lenaerts (UHasselt)

presenting joined work with

Dr. Florian Allroggen (MIT) \& Prof. Robert Malina (UHasselt)

$\rightarrow$

UHASSELT 


\section{Aims}

1. Discuss relationship between transport and geography

2. Definition and operationalisation of relevant metrics

3. Comparison with typical impact metrics 


\section{Economic Geography}

\section{New Economic Geography}

- $\quad$ Started in 1991 by P. Krugman

- Introduction of space and geography to economics and economic modelling

- 2008 Noble Prize on "location of economic activity"

- Empirical counterpart: Spatial Econometrics

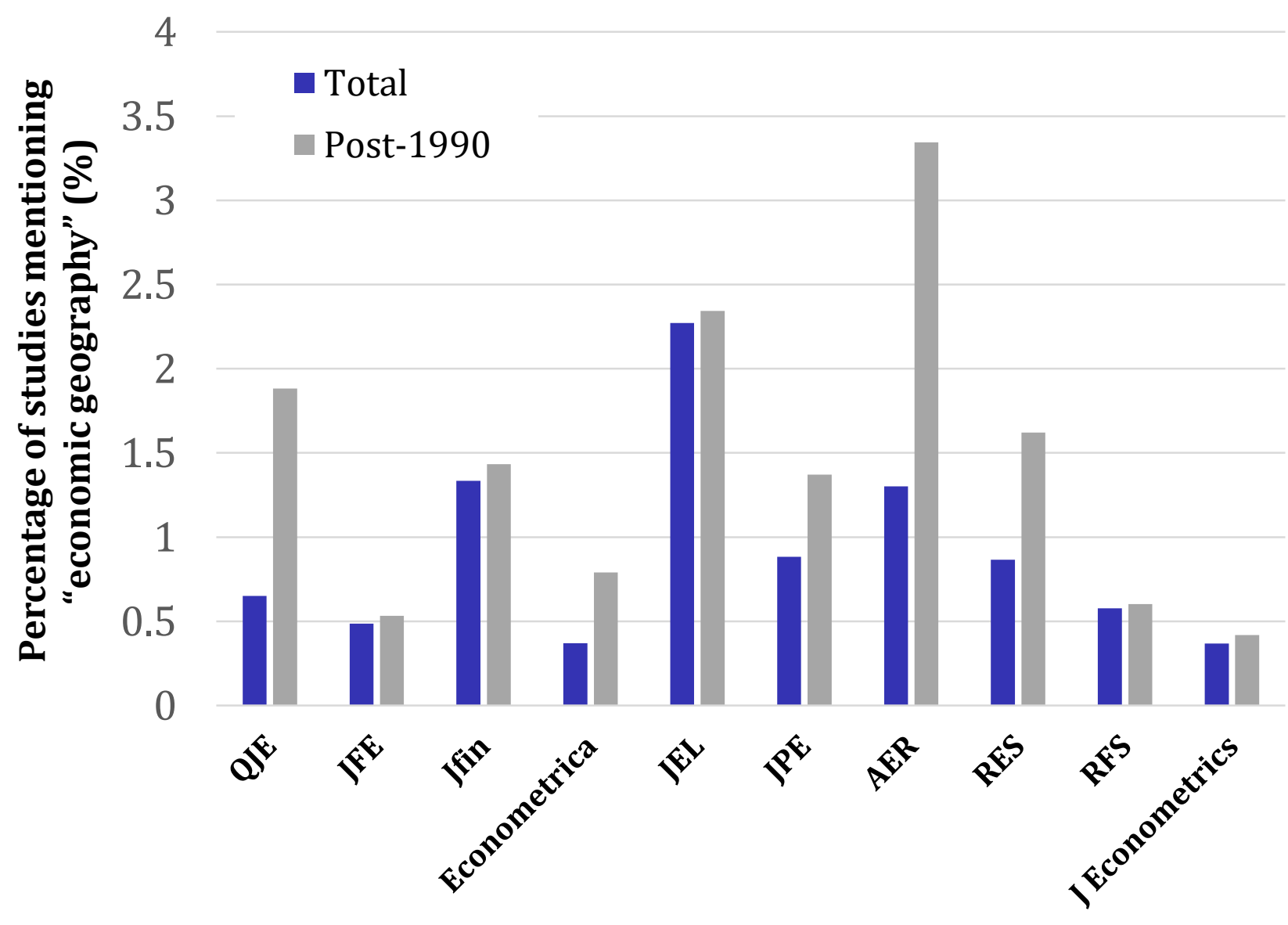




\section{Remoteness and Transportation}
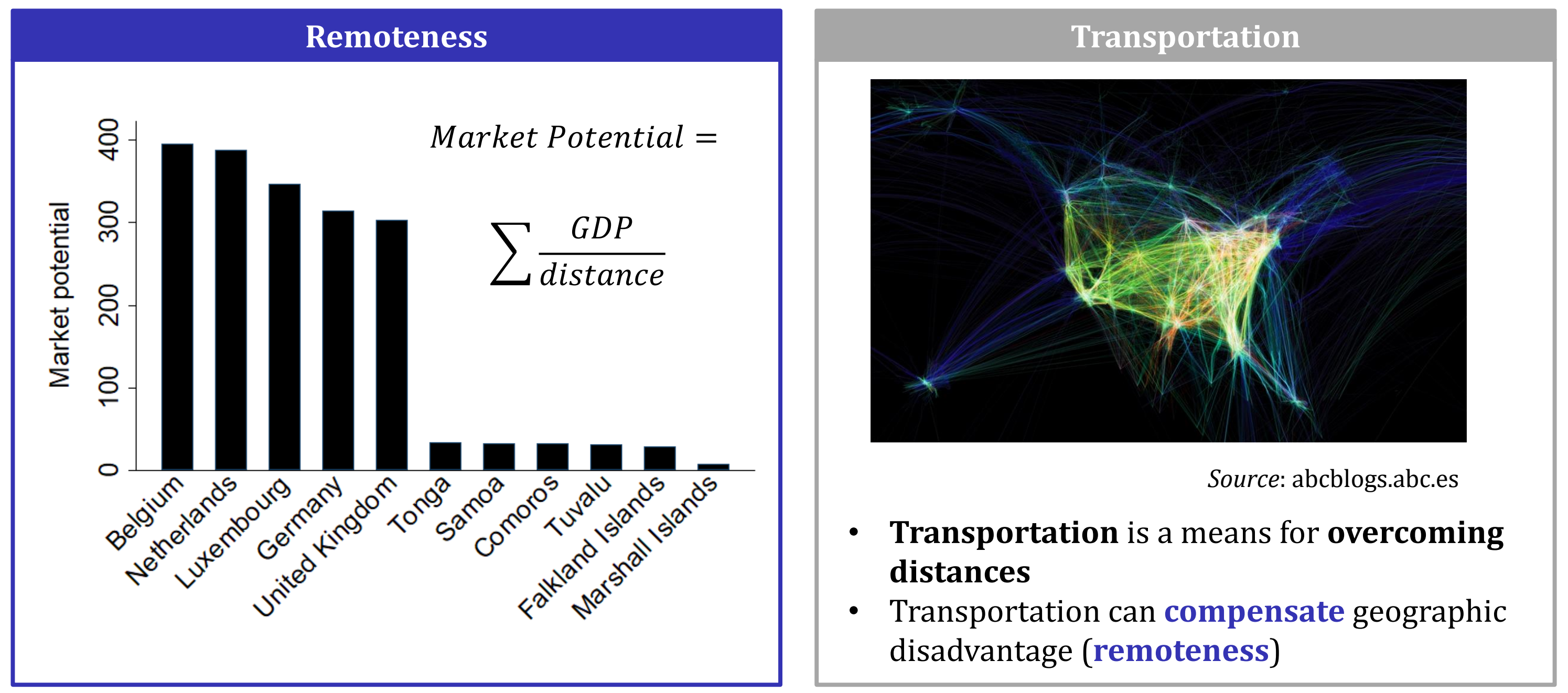


\section{Different types of transfer cost}

Type of interaction cost

Trade and investment cost

Transaction and supply
Cost determinants

(physical and economic distance)

Institutional and policy barriers

Borders

Geographic contiguity

Time differences

Information and communication infrastructure and services Technological barriers

Cultural barriers (especially language)

\section{Distance}

Transportation infrastructure and services 


\section{Transportation cost}

\section{Direct (monetary) costs}

Ticket fare

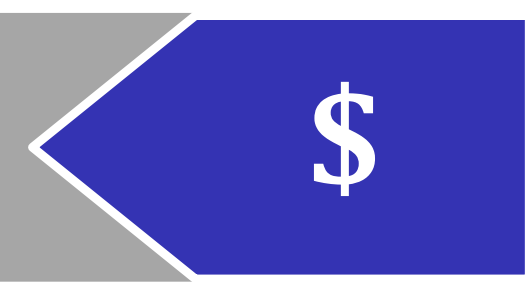

\section{Opportunity costs}

Travel and waiting time

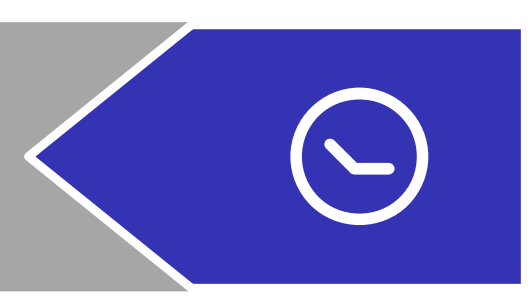

\section{Disutility costs}

Risk premiums, Level of comfort, Option values, Travel adjustment

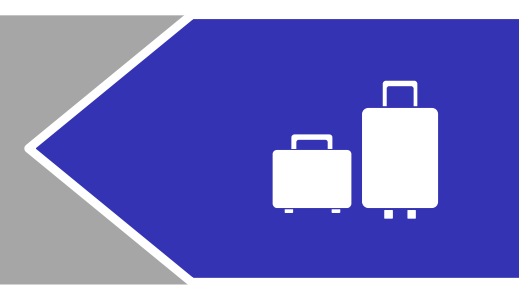

Example: cost of air freight

$\%$ of value shipped

15

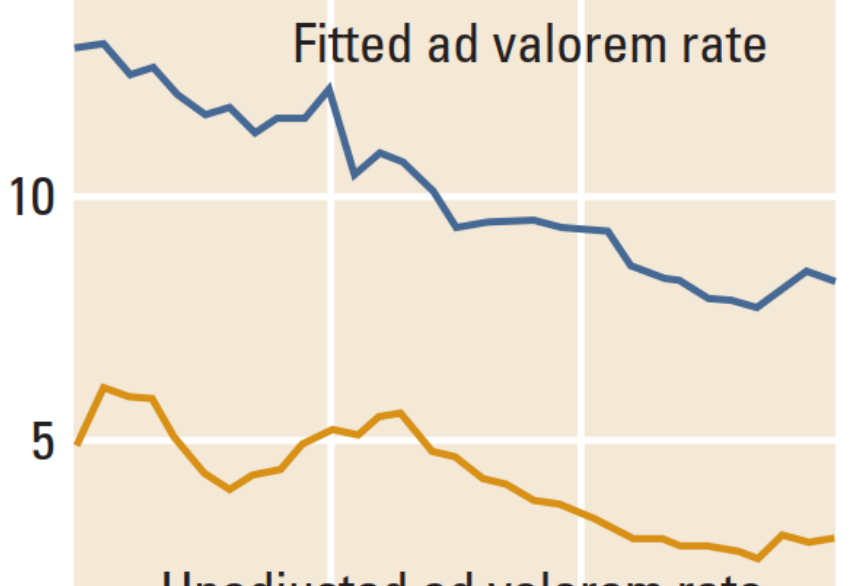

Unadjusted ad valorem rate

0

1974

1984

1994

2004

Source: Hummels (2007) 


\section{Interaction potential: a simplified framework}

(1) Pull by opportunities at remote markets

\section{Home region}

Consumers, Producers, Suppliers, Workers

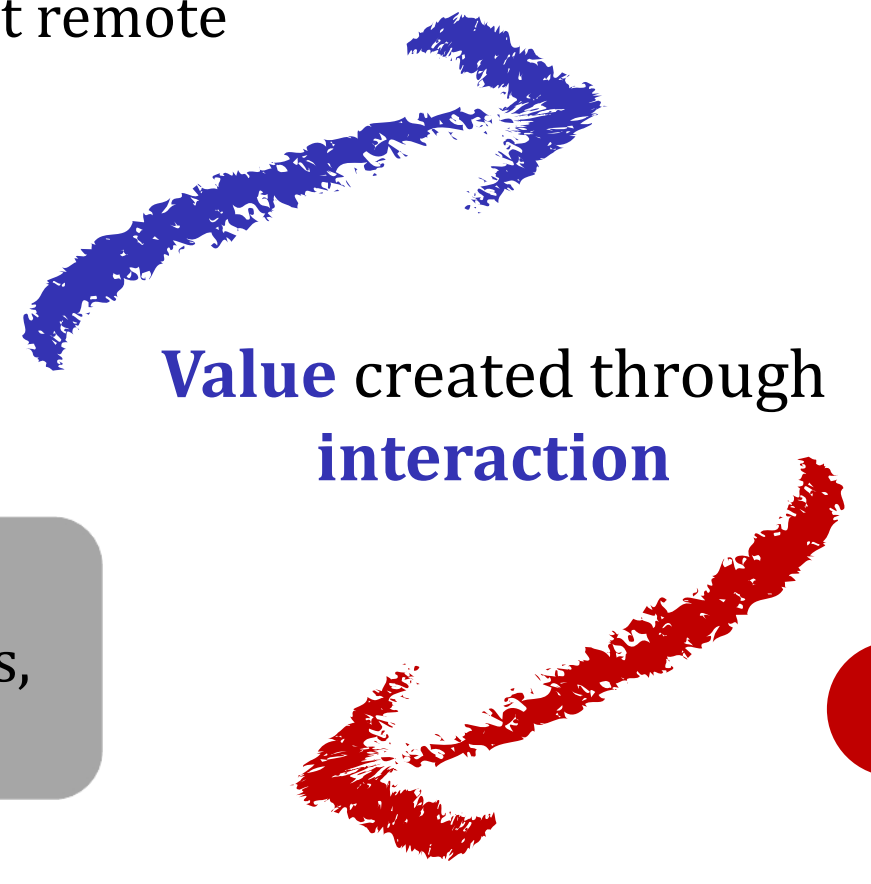

Remote Markets

Consumers, Producers, Suppliers, Workers

2 Push by cost of overcoming frictions (distance and geography) 


\section{Interaction potential: a simplified framework}

(1) Pull by opportunities at remote markets

Home region

Consumers, Producers, Suppliers, Workers
Remote Markets

Consumers, Producers, Suppliers, Workers

2 Push by cost of overcoming frictions (distance and geography)

\section{Transport Component}




\section{Accessibility}

\section{Definition}

Negative function of transfer cost to, and a positive function of the market value of all destinations

\section{Transport component}

- Generalized travel cost

- Geography

- Services and infrastructure

- Passenger and freight travel travel demand

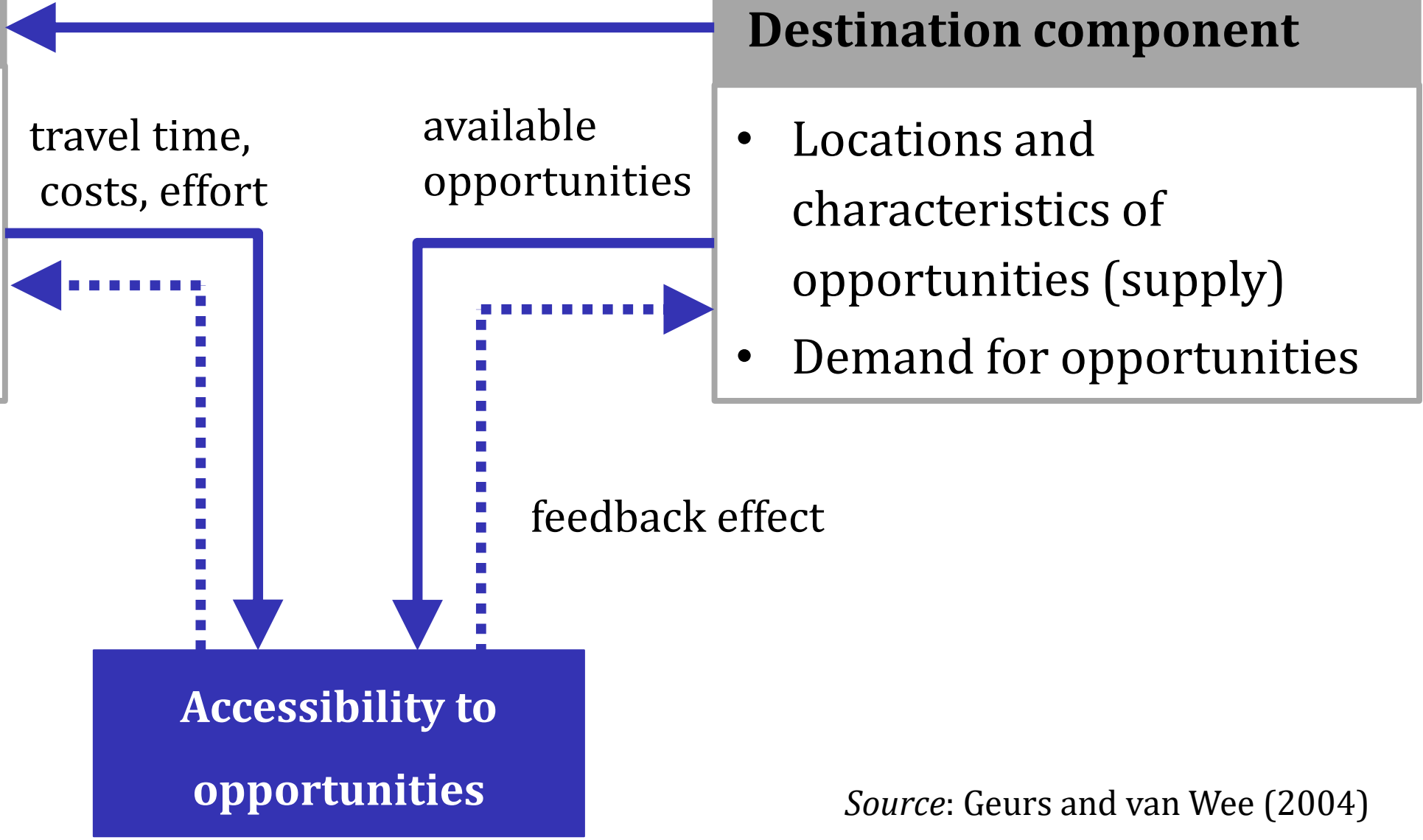




\section{Connectivity}

\section{Definition}

Negative function of transfer cost between nodes in an aspatial network, and a positive function of the market value of all destinations excluding the home market

\section{Transport component}

- Distance-independent cost

- Services offerings and quality

- Passenger and freight travel travel demand

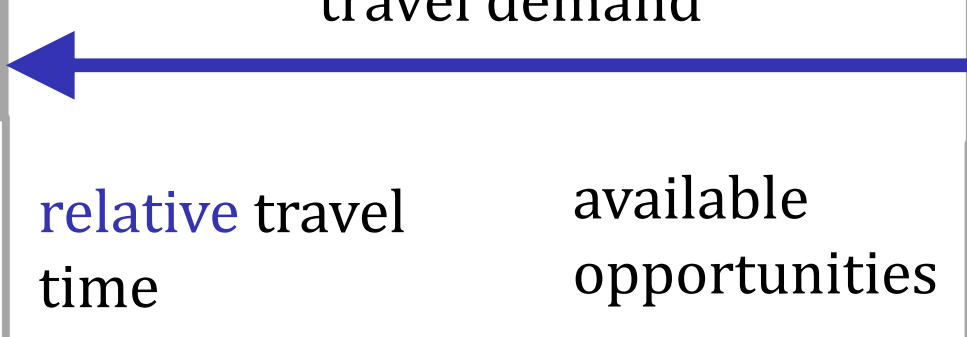

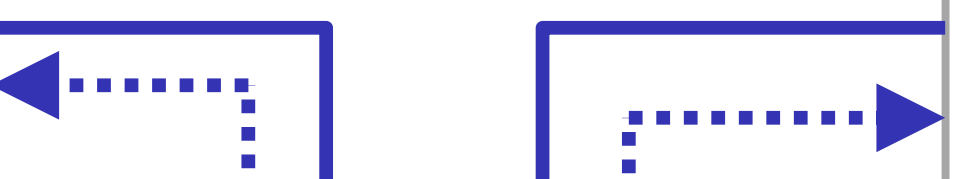

\section{Destination component}

- Locations and characteristics of opportunities (supply)

- Demand for opportunities

Connectivity to 


\section{Summary: Decomposition of transport concepts}

\begin{tabular}{|c|c|c|c|}
\hline & \multicolumn{2}{|c|}{ Weighting of links } \\
\hline & & $\begin{array}{l}\text { Weighted } \\
\text { (destination quality) }\end{array}$ & $\begin{array}{l}\text { Invariant } \\
\text { (no destination quality) }\end{array}$ \\
\hline \multirow{2}{*}{ 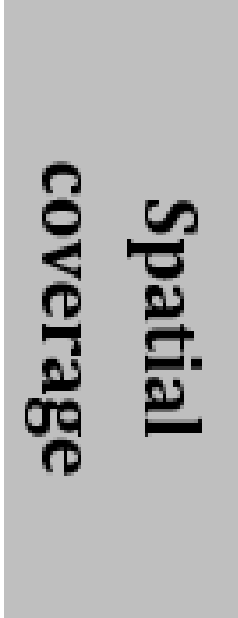 } & $\begin{array}{l}\text { Spatial } \\
\text { (generalized } \\
\text { transportation costs) }\end{array}$ & Accessibility & $\begin{array}{c}\text { Destination-invariant } \\
\text { Accessibility }\end{array}$ \\
\hline & $\begin{array}{l}\text { Aspatial } \\
\text { (service and } \\
\text { infrastructure quality) }\end{array}$ & Market Connectivity & Connectivity \\
\hline
\end{tabular}


Accessibility and Connectivity metrics 


\section{Operationalizing air transport metrics}

\begin{tabular}{lll}
\hline Metric type & Subfamily & Definition \\
\hline & $\begin{array}{l}\text { Centrality- } \\
\text { type }\end{array}$ & $\begin{array}{l}\text { Number of connected } \\
\text { airports, counted as } \\
\text { the airports } \\
\text { themselves or the } \\
\text { steps on best routings } \\
\text { only }\end{array}$ \\
\cline { 2 - 3 } $\begin{array}{l}\text { Connectivity- } \\
\text { type } \\
\text { (Aspatial) }\end{array}$ & $\begin{array}{l}\text { Feasible } \\
\text { links }\end{array}$ & $\begin{array}{l}\text { Number of feasible } \\
\text { connections for all } \\
\text { routings }\end{array}$ \\
\cline { 2 - 3 } & Link & $\begin{array}{l}\text { Number of weighted } \\
\text { connections for all } \\
\text { routings }\end{array}$ \\
\hline Accessibility-type (Spatial) & $\begin{array}{l}\text { Measures capturing } \\
\text { generalized air } \\
\text { transportation costs }\end{array}$ \\
\hline
\end{tabular}




\section{Link quality}

\begin{tabular}{|c|c|c|c|c|}
\hline \multirow{2}{*}{ Metric type } & \multirow{2}{*}{ Subfamily } & \multirow{2}{*}{ Definition } & \multicolumn{2}{|c|}{ Examples } \\
\hline & & & Market & Non-market \\
\hline \multirow{3}{*}{$\begin{array}{l}\text { Connectivity- } \\
\text { type } \\
\text { (Aspatial) }\end{array}$} & $\begin{array}{l}\text { Centrality- } \\
\text { type }\end{array}$ & $\begin{array}{l}\text { Number of connected } \\
\text { airports, counted as } \\
\text { the airports } \\
\text { themselves or the } \\
\text { steps on best routings } \\
\text { only }\end{array}$ & $\begin{array}{l}\text { Cristea } \\
\text { and Danila }\end{array}$ & $\begin{array}{l}\text { Closeness, } \\
\text { degreeness, } \\
\text { gross vertex }\end{array}$ \\
\hline & $\begin{array}{l}\text { Feasible } \\
\text { links }\end{array}$ & $\begin{array}{l}\text { Number of feasible } \\
\text { connections for all } \\
\text { routings }\end{array}$ & & $\begin{array}{l}\text { Doganis, } \\
\text { Seredyński }\end{array}$ \\
\hline & $\begin{array}{l}\text { Link } \\
\text { quality }\end{array}$ & $\begin{array}{l}\text { Number of weighted } \\
\text { connections for all } \\
\text { routings }\end{array}$ & & $\begin{array}{l}\text { Bootsma, } \\
\text { Danesi, WNX, } \\
\text { Netscan, CCI, } \\
\text { Jenkins }\end{array}$ \\
\hline \multicolumn{2}{|c|}{ Accessibility-type (Spatial) } & $\begin{array}{l}\text { Measures capturing } \\
\text { generalized air } \\
\text { transportation costs }\end{array}$ & $\begin{array}{l}\text { ACI, PATH } \\
\text { theorem }\end{array}$ & $\begin{array}{l}\text { Quickest path } \\
\text { length }\end{array}$ \\
\hline
\end{tabular}

\section{Airport Connectivity Quality Index (ACQI)}

- Wittman and Swelbar (2013)

- Market connectivity link quality-type

- $C O N=\sum_{r} \delta_{r} \cdot w_{d_{r}}+\alpha \sum_{r^{\prime}} \delta_{r^{\prime}} \cdot w_{d_{r^{\prime}}}$ with $\delta_{r}=\left\{\begin{array}{l}1 \text { if direct flight } \\ 0 \text { else }\end{array}\right.$ and analogue for onestop connections $\left(r^{\prime}\right)$

- Destination weighting based on socioeconomic importance of each destination proxied by hub status. 


\section{Link quality}

\begin{tabular}{|c|c|c|c|c|}
\hline \multirow{2}{*}{ Metric type } & \multirow{2}{*}{ Subfamily } & \multirow{2}{*}{ Definition } & \multicolumn{2}{|c|}{ Examples } \\
\hline & & & Market & Non-market \\
\hline \multirow{3}{*}{$\begin{array}{l}\text { Connectivity- } \\
\text { type } \\
\text { (Aspatial) }\end{array}$} & $\begin{array}{l}\text { Centrality- } \\
\text { type }\end{array}$ & $\begin{array}{l}\text { Number of connected } \\
\text { airports, counted as } \\
\text { the airports } \\
\text { themselves or the } \\
\text { steps on best routings } \\
\text { only }\end{array}$ & $\begin{array}{l}\text { Cristea } \\
\text { and Danila }\end{array}$ & $\begin{array}{l}\text { Closeness, } \\
\text { degreeness, } \\
\text { gross vertex }\end{array}$ \\
\hline & $\begin{array}{l}\text { Feasible } \\
\text { links }\end{array}$ & $\begin{array}{l}\text { Number of feasible } \\
\text { connections for all } \\
\text { routings }\end{array}$ & & $\begin{array}{l}\text { Doganis, } \\
\text { Seredyński }\end{array}$ \\
\hline & $\begin{array}{l}\text { Link } \\
\text { quality }\end{array}$ & $\begin{array}{l}\text { Number of weighted } \\
\text { connections for all } \\
\text { routings }\end{array}$ & $\begin{array}{l}\text { ACQI, } \\
\text { GCI }\end{array}$ & $\begin{array}{l}\text { Bootsma, } \\
\text { Danesi, WNX, } \\
\text { Netscan, CCI, } \\
\text { Jenkins }\end{array}$ \\
\hline \multicolumn{2}{|c|}{ Accessibility-type (Spatial) } & $\begin{array}{l}\text { Measures capturing } \\
\text { generalized air } \\
\text { transportation costs }\end{array}$ & $\begin{array}{l}\text { ACI, PATH } \\
\text { theorem }\end{array}$ & $\begin{array}{l}\text { Quickest path } \\
\text { length }\end{array}$ \\
\hline
\end{tabular}

\section{Netscan}

- Veldhuis (1997) \& De Wit et al. (2009)

- Non-market connectivity link quality-type

- Perceived travel time

- Excess travel time

- $C O N=\sum_{r} \alpha_{r} \cdot f_{r}$

$>\alpha_{r}: f($ Excess travel time $) \rightarrow[0,1]$

$>f_{r}$ : frequency 


\section{Link quality}

\begin{tabular}{|c|c|c|c|c|}
\hline \multirow{2}{*}{ Metric type } & \multirow{2}{*}{ Subfamily } & \multirow{2}{*}{ Definition } & \multicolumn{2}{|c|}{ Examples } \\
\hline & & & Market & Non-market \\
\hline \multirow{3}{*}{$\begin{array}{l}\text { Connectivity- } \\
\text { type } \\
\text { (Aspatial) }\end{array}$} & $\begin{array}{l}\text { Centrality- } \\
\text { type }\end{array}$ & $\begin{array}{l}\text { Number of connected } \\
\text { airports, counted as } \\
\text { the airports } \\
\text { themselves or the } \\
\text { steps on best routings } \\
\text { only }\end{array}$ & $\begin{array}{l}\text { Cristea } \\
\text { and Danila }\end{array}$ & $\begin{array}{l}\text { Closeness, } \\
\text { degreeness, } \\
\text { gross vertex }\end{array}$ \\
\hline & $\begin{array}{l}\text { Feasible } \\
\text { links }\end{array}$ & $\begin{array}{l}\text { Number of feasible } \\
\text { connections for all } \\
\text { routings }\end{array}$ & & $\begin{array}{l}\text { Doganis, } \\
\text { Seredyński }\end{array}$ \\
\hline & $\begin{array}{l}\text { Link } \\
\text { quality }\end{array}$ & $\begin{array}{l}\text { Number of weighted } \\
\text { connections for all } \\
\text { routings }\end{array}$ & & $\begin{array}{l}\text { Bootsma, } \\
\text { Danesi, WNX, } \\
\text { Netscan, CCI, } \\
\text { Jenkins }\end{array}$ \\
\hline \multicolumn{2}{|c|}{ Accessibility-type (Spatial) } & $\begin{array}{l}\text { Measures capturing } \\
\text { generalized air } \\
\text { transportation costs }\end{array}$ & $\begin{array}{l}\text { ACI, PATH } \\
\text { theorem }\end{array}$ & $\begin{array}{l}\text { Quickest path } \\
\text { length }\end{array}$ \\
\hline
\end{tabular}

\section{Global Connectivity Index (GCI)}

- Allroggen et al. (2015)

- Market connectivity link quality-type

- Perceived travel time

- Detour factor

- $\operatorname{CON}=\sum_{r} \alpha_{r} \cdot f_{r} \cdot w_{d_{r}}$

$>\alpha_{r}: f$ (Detour factor $) \rightarrow[0,1]$

$>f_{r}:$ frequency

$>w_{d_{r}}$ : Destination weighing based on population and income 


\section{Link quality}

\begin{tabular}{|c|c|c|c|c|}
\hline \multirow{2}{*}{ Metric type } & \multirow{2}{*}{ Subfamily } & \multirow{2}{*}{ Definition } & \multicolumn{2}{|c|}{ Examples } \\
\hline & & & Market & Non-market \\
\hline \multirow{3}{*}{$\begin{array}{l}\text { Connectivity- } \\
\text { type } \\
\text { (Aspatial) }\end{array}$} & $\begin{array}{l}\text { Centrality- } \\
\text { type }\end{array}$ & $\begin{array}{l}\text { Number of connected } \\
\text { airports, counted as } \\
\text { the airports } \\
\text { themselves or the } \\
\text { steps on best routings } \\
\text { only }\end{array}$ & $\begin{array}{l}\text { Cristea } \\
\text { and Danila }\end{array}$ & $\begin{array}{l}\text { Closeness, } \\
\text { degreeness, } \\
\text { gross vertex }\end{array}$ \\
\hline & $\begin{array}{l}\text { Feasible } \\
\text { links }\end{array}$ & $\begin{array}{l}\text { Number of feasible } \\
\text { connections for all } \\
\text { routings }\end{array}$ & & $\begin{array}{l}\text { Doganis, } \\
\text { Seredyński }\end{array}$ \\
\hline & $\begin{array}{l}\text { Link } \\
\text { quality }\end{array}$ & $\begin{array}{l}\text { Number of weighted } \\
\text { connections for all } \\
\text { routings }\end{array}$ & $\begin{array}{l}\text { ACQI, } \\
\text { GCI }\end{array}$ & $\begin{array}{l}\text { Bootsma, } \\
\text { Danesi, WNX, } \\
\text { Netscan, CCI, } \\
\text { Jenkins }\end{array}$ \\
\hline \multicolumn{2}{|c|}{ Accessibility-type (Spatial) } & $\begin{array}{l}\text { Measures capturing } \\
\text { generalized air } \\
\text { transportation costs }\end{array}$ & theo & $\begin{array}{l}\text { Quickest path } \\
\text { length }\end{array}$ \\
\hline
\end{tabular}

\section{Path aggregation theorem (PATH)}

- Mandel et al. (2017)

- Extension of Bootsma connectivity

- Additional weights based on OD path utilities (path choice model)

- No a priori quality weights assumptions but passenger preferences, incl. ticket prices 


\section{Implications for impact assessment}




\section{Empirical evidence on the economic impacts of aviation}

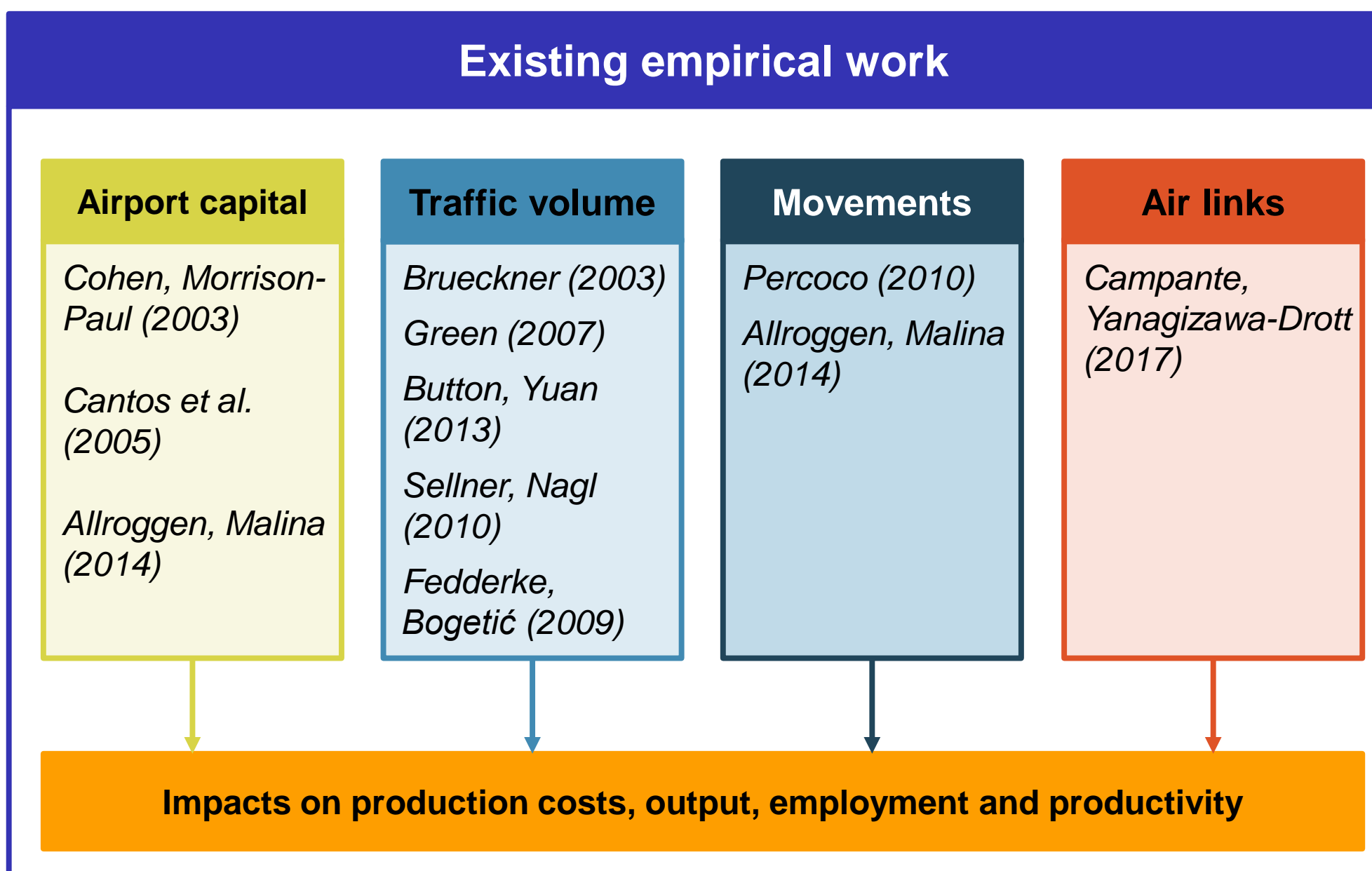




\section{Connectivity vs Departures}
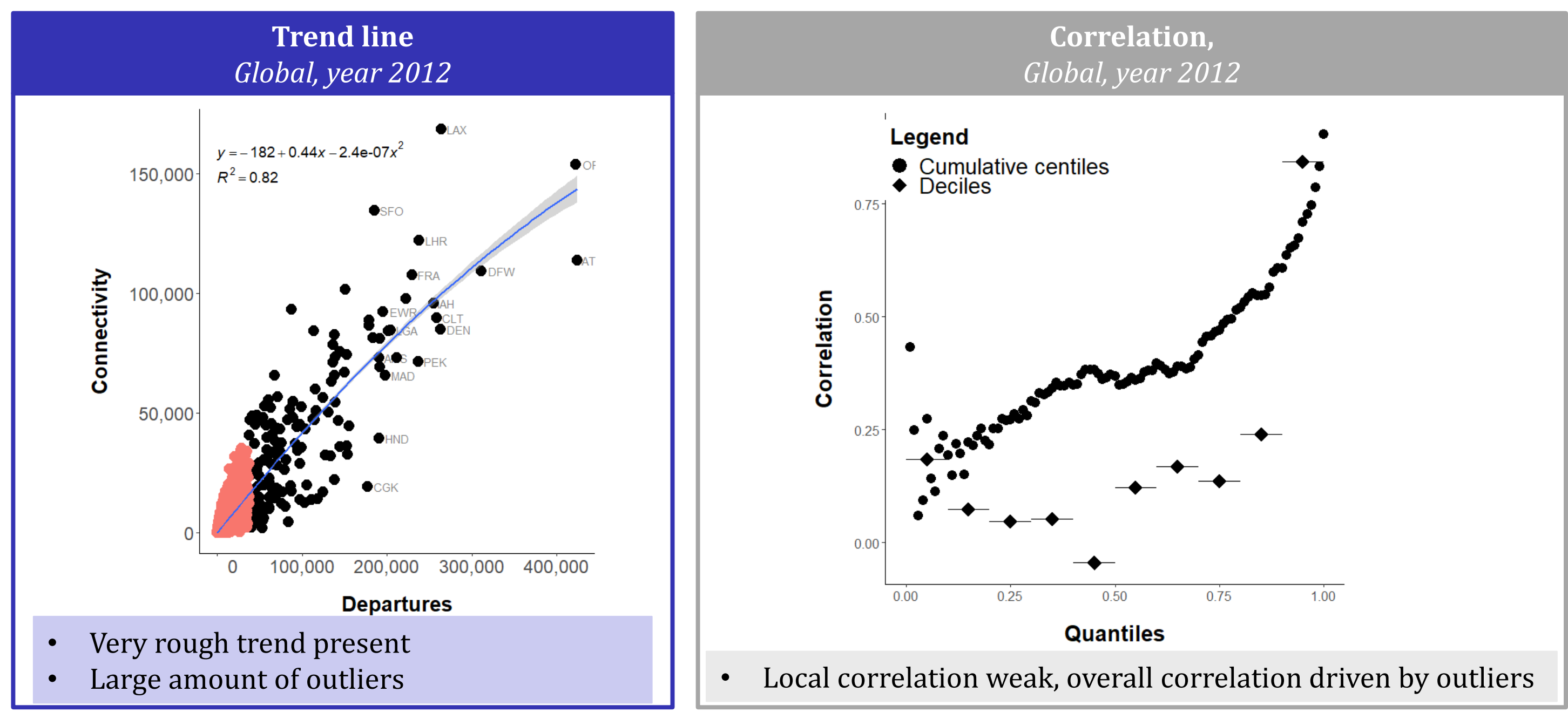


\section{Connectivity vs Departures (2)}

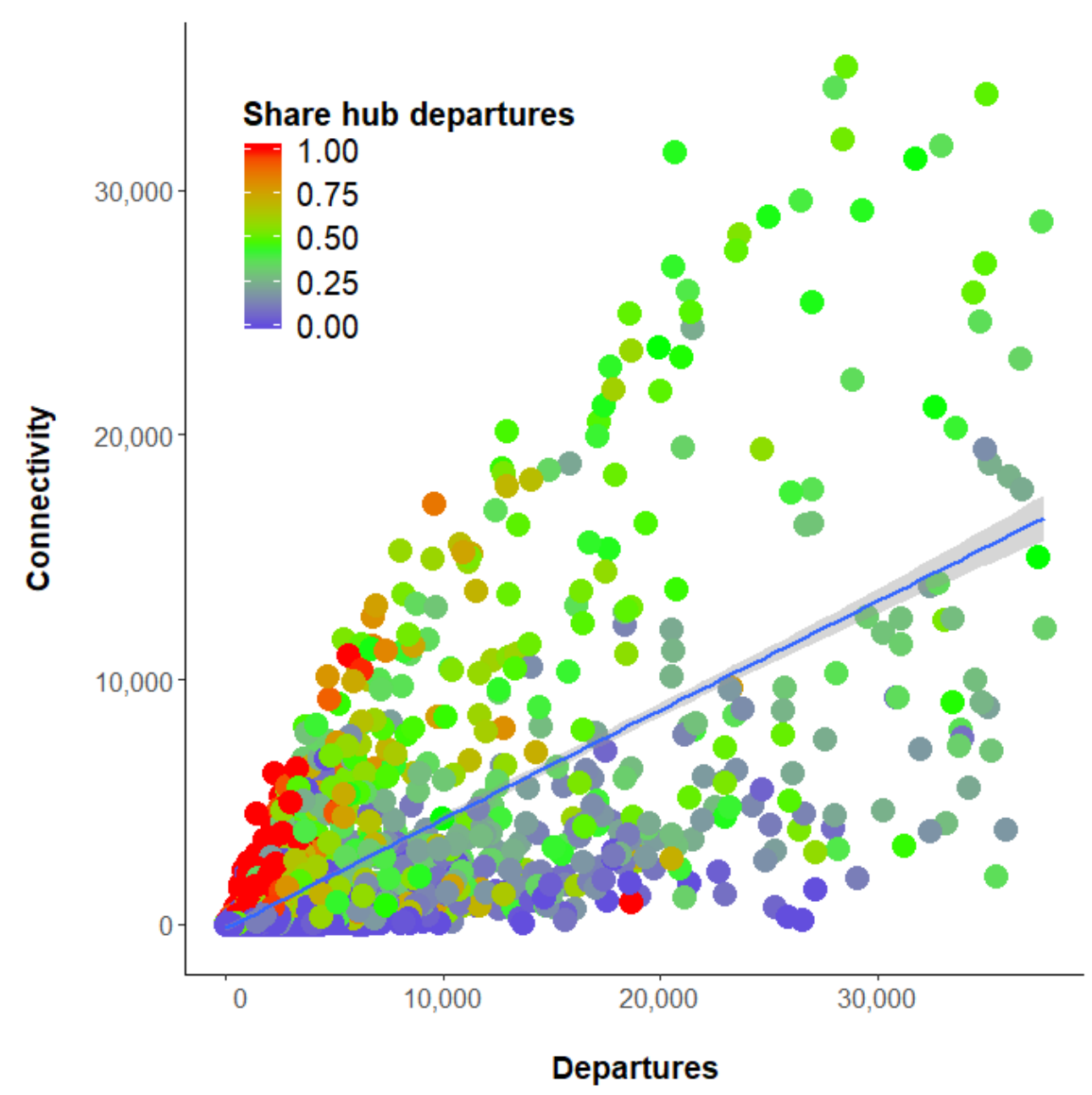

\section{Network structure}

- Traffic statistics do not capture network structure

- Importance of indirect connections: many small airports rely on onward connectivity

- Connectivity metrics needed to value access through network 


\section{Conclusions}

- Connectivity and accessibility are essential for measuring and understanding the benefits of air transport

- Connectivity: aspatial cost

- Measures level of services offerings and quality

- Determines transportation's ability to overcome distance

- Market potential: (purely) spatial cost

- Measures remoteness/centrality

- Accessibility: overall cost

- Measures (net) interaction cost, but no separation between transport and space

- Determines geographical distribution of economic activity

- Dilemma between accessibility and connectivity/market potential 


\section{Centre for Environmental Sciences}

UHasselt

Bert Lenaerts

bert.lenaerts@uhasselt.be

Robert Malina

robert.malina@uhasselt.be
Florian Allroggen

fallrogg@mit.edu

UHASSELT 